\title{
Optimización del Transporte de Caña de Azúcar en Tucumán utilizando Sistemas Multiagentes
}

\author{
Nicolás Majorel Padilla ${ }^{1,2}$, Agustín Décima ${ }^{1}$, Adrián Will ${ }^{1,3}$, Sebastián Rodríguez ${ }^{1}$ y \\ Oscar Diez ${ }^{4}$ \\ ${ }^{1}$ CITAT, FRT, UTN, Tucumán, Argentina \\ \{nicolas.majorel, agustin.decima, adrian.will, \\ sebastian.rodriguez\}@citat.org.ar, \\ ${ }^{2}$ DEEC, FACET, UNT, Tucumán, Argentina \\ ${ }^{3}$ Departamento de Matemática, FACET, UNT, Tucumán, Argentina \\ ${ }^{4}$ EEAOC, Tucumán, Argentina \\ osandiez@eeaoc.org.ar
}

\begin{abstract}
Resumen. El presente trabajo pretende mostrar el desarrollo de un modelo matemático y un Sistema Multiagentes para la optimización del transporte de caña de azúcar, desde el productor cañero hasta el ingenio. Llevar a cabo esta optimización implica considerar factores tales como la eficiencia de producción de caña, el rendimiento de los ingenios, los costos de transporte, etc., que hacen de la misma un problema complejo. Nuestra propuesta intenta maximizar el balance energético del sistema, teniendo en cuenta varias restricciones operativas, buscando un sistema escalable y paralelizable debido al tamaño y complejidad del problema. El sistema se prueba con datos sintéticos, y se compara con otro similar basado en Programación Lineal desarrollado en trabajos anteriores. Los resultados muestran que la herramienta es adecuada para el sistema planteado, obteniendo resultados similares en mucho menos tiempo.
\end{abstract}

Palabras clave. Optimización, transporte, caña de azúcar, sistemas multiagentes, fuerzas de atracción.

\section{$1 \quad$ Introducción}

La provincia de Tucumán es la principal productora argentina de caña de azúcar, con el $66 \%$ de la producción nacional. Es una de las principales actividades de la provincia, estimándose en un $10.5 \%$ del PBI provincial, lo que representa aproximadamente unos 1500 millones de pesos [1]. Además, la agroindustria azucarera representa un $42 \%$ del sector de la industria manufacturera de la provincia [2].

A pesar de la importancia económica de esta actividad para la provincia, en la actualidad la plantación, cosecha y transporte no presenta una planificación optimizada. Esta situación, agravada por otros elementos socio-económicos (mal mantenimiento de los vehículos, etc.), reduce claramente la rentabilidad de la actividad tanto para productores como para los ingenios. El transporte de caña de azúcar desde los produc- 
tores en el campo hasta los ingenios para su procesamiento es un problema complejo, de gran tamaño, donde intervienen gran cantidad de variables. Una solución realista para el problema requiere tener en cuenta muchos aspectos diferentes y restricciones a respetar.

Actualmente, durante los primeros y los últimos días de la zafra (cosecha temprana y cosecha tardía), la caña madura no es suficiente para satisfacer la demanda de los ingenios. Inversamente, en el período óptimo (aproximadamente Junio a Agosto) la cantidad de caña sobrepasa la capacidad de procesamiento. Esta situación impacta fuertemente tanto a los productores como a los ingenios, ya que genera problemas a los productores para cosechar y entregar en el período óptimo, y a los ingenios porque resulta complicado cumplir sus cuotas de producción en los períodos temprano y tardío. En este contexto una planificación apropiada de los transportes y tiempos de siembra y cosecha pueden transformarse en un gran beneficio para todo el sistema productivo.

Más aún, el aspecto negativo de esta situación no se reduce a lo económico ya que impacta en el medio ambiente (recorridos extensos con vehículos mal mantenidos, excesivamente cargados, etc.), y seguridad vial (aumento del tráfico pesado a baja velocidad en rutas altamente transitadas), entre otros. Un sistema unificado permitiría ordenar el movimiento de la producción dando como resultado un cronograma de cosechas y entregas, respetando todas las restricciones planteadas, y sugiriendo puntos de la red vial que deberían ser especialmente monitoreados y mantenidos.

Otro aspecto del problema a tener en cuenta, es la iniciativa actual para incentivar la producción de Biocombustibles, especialmente Bioetanol. La sustitución de hasta $5 \%$ de alcohol en las naftas, medida que por ley comenzó a aplicarse este año en nuestro país, muestra la importancia de optimizar los consumos de combustibles fósiles en la producción del Bioetanol, a fin de maximizar esta sustitución.

Es importante destacar que, dado el gran volumen de caña de azúcar que se transporta por año dentro de la provincia de Tucumán (aproximadamente 1400 millones de toneladas), cualquier mejora que se logre puede resultar en grandes impactos, tanto en consumo de combustible, medio ambiente, economía, y los diversos aspectos logísticos y de producción relacionados a la industria del azúcar de caña.

El presente trabajo representa el primer paso de un proyecto a largo plazo para la optimización del transporte de caña de azúcar en la provincia de Tucumán. Se tomó la decisión de abordar en esta primera etapa el problema de entrega de la cosecha para un solo día (esto es, tomando como dato la cantidad y posición de las parcelas cosechadas). Esto se hace por razones tanto matemáticas como industriales: por un lado permite ajustar el modelo matemático utilizado y estudiar la performance de los diferentes algoritmos, y por otro lado resulta de interés especial para ingenios y cañeros, ya que normalmente las condiciones cambiantes de la cosecha hacen más práctica esta aproximación (una lluvia inesperada, helada, $u$ otros eventos extremos pueden alterar las condiciones en gran medida, creando la necesidad de obtener una nueva solución de forma inmediata).

Este problema es sólo parte de otro mayor, relacionado con la eficiencia global del sistema productivo, ya que existen numerosos casos de industrias en el país, cuya materia prima proviene del campo y es transportada hacia los centros de producción 
utilizando la red vial existente en todo tipo de vehículos y sin ninguna planificación, ocasionando problemas similares (accidentes de tránsito, gastos excesivos de combustible por falta de planificación, pérdidas de calidad en materia prima por demoras, etc.).

La propuesta consiste en buscar una solución al problema planteado utilizando Sistemas Multiagentes, comparando los resultados obtenidos con los logrados previamente en [3], donde se utiliza una propuesta basada en programación lineal.

El trabajo está organizado de la siguiente manera: en la sección siguiente se presenta el estado del arte, en la sección 3 se describe la propuesta de trabajo y los modelos matemáticos asociados, en la sección 4 se presentan los resultados obtenidos y finalmente en la sección 5 se presentan las conclusiones del trabajo.

\section{Estado del arte}

El problema de logística de productos agroindustriales es un problema de larga data, y se encuentra presente en todas las industrias del mundo que utilicen como materia prima productos agroindustriales. En el caso de la caña de azúcar, resulta susceptible a deterioro por estacionamiento por lo que las demoras en estacionamiento son particularmente preocupantes. Se realizaron experiencias en el tema en Australia, Sudáfrica, Brasil, Cuba, y Tailandia [4-9]. Es importante destacar que algunas experiencias resultaron exitosas y se vienen aplicando desde hace varios años (Australia y Sudáfrica), y en otros casos se encuentran en progreso (Cuba, Brasil y Tailandia). Se inscribe dentro del contexto mayor de Supply Chain Management, del cual existen numerosos trabajos y herramientas utilizadas [10-16].

Como principales herramientas utilizadas, se cuentan los sistemas Multiagentes (particularmente adaptados a problemas de tráfico [12-15]), los Algoritmos Genéticos [10-11], y algoritmos más tradicionales pero no menos efectivos provenientes de Investigación Operativa y Programación Lineal [9]. Es importante destacar que existe una variedad de herramientas tanto analíticas como heurísticas que permiten resolver este problema, cada una con diferentes características, ventajas y desventajas (especialmente la cantidad y tipo de restricciones operativas que puede manejar, y el tamaño de problemas para los cuales resultan eficientes). También se debe destacar que un sistema de este tipo para ser efectivo, debe adecuarse a la realidad y características propias del lugar donde se aplica (red vial, industrias, productores, clima, situación económica, etc.).

\section{Propuesta}

\subsection{Definiciones}

Se divide la zona productora de caña de azúcar en unidades productivas de la misma superficie, denominadas parcelas. Cada parcela produce una cantidad de caña que puede variar en cantidad y calidad, dependiendo de diversos factores. Con esta divi- 
sión, se estima que en toda la provincia se cuenta con una cantidad total aproximada de 300.000 parcelas.

Por otra parte, cada ingenio (fábrica de azúcar, en nuestro país a partir de caña) demanda un cierto número diario de toneladas de caña para ser procesadas, y tiene cierta eficiencia en este procesamiento de acuerdo a la tecnología con la que cuenta. En la actualidad, la provincia de Tucumán cuenta con 15 ingenios.

Luego de estudios preliminares del problema, se decidió utilizar la energía como variable principal de la optimización, en contraposición a otras posibilidades como basarla en costos económicos. Esto presenta la ventaja de ser imparcial y posee menos sesgo hacia los ingenios más grandes (y por lo tanto de mayor poder adquisitivo), sino que premia la eficiencia y no permite grandes recorridos debido al gasto de combustible (que en algunos casos puede ser compensado por ganancias económicas). El balance energético (de ahora en más $\mathrm{BE}$ ) se define como el cociente entre la energía total obtenida como producto final (sea en forma de azúcar o de alcohol) y la energía total insumida a lo largo del proceso productivo (sea en forma de fertilizantes, combustible utilizado, pérdidas de materia prima por estacionamiento, etc.). Particularizando este concepto para las parcelas, el BE es el cociente entre la energía obtenida (en forma de toneladas de caña) y la energía insumida en la siembra, crecimiento y cosecha de la misma. Este concepto permite comparar distintas parcelas entre sí, resultando más eficiente aquella parcela que presenta un mayor BE. En el caso de los ingenios, cuanto mayor sea su BE, más eficiente será (ya que extrae mayor cantidad de energía para igual energía invertida, u obtiene la misma energía final con menor inversión).

El presente trabajo aborda sólo el problema de la cosecha para un día fijo (o sea, tomando como dato la cantidad y posición de parcelas que se cosechan). Esta decisión fue tomada en conjunto con la parte industrial del problema, ya que entre otras razones, es usual que condiciones imprevistas como heladas, cortes de ruta o lluvias excesivas, modifiquen sustancialmente el escenario y obliguen a conseguir una solución al problema de manera inmediata, para las nuevas condiciones. De esta manera, se toman como datos la producción de caña de las parcelas (número de parcelas, posición, características de su producción) y la demanda de los ingenios para el día en cuestión.

Se tienen entonces los siguientes datos:

- Un conjunto P de $m$ parcelas, cada una con su valor de producción (medida en toneladas de caña) y su eficiencia, indicada por su balance energético. Este último valor es siempre mayor que 1 , ya que se obtiene mas energía de la que se invierte (no se considera la energía del sol en el cálculo).

- Un conjunto I de $n$ ingenios, cada uno de ellos con su demanda máxima diaria (medida en toneladas de caña), y su eficiencia, indicada por su balance energético. Este último valor es siempre menor que1, ya que se obtiene menos energía de la que ingresa.

- Una matriz de costos $\mathrm{C}$, de tamaño $m \times n$, en la cual cada valor $c_{i j}$ expresa el costo (medido como balance energético) de transportar la producción desde la parcela $i$ hasta el ingenio $j$. Este costo incluye todos los costos traducidos a energía, desde 
combustible necesario para el transporte, pérdidas estimadas por mal estado del camino, pérdidas por estacionamiento, mantenimiento de los vehículos, etc.).

El objetivo del presente trabajo consiste entonces en encontrar una distribución de las $m$ parcelas en los $n$ ingenios de manera que se cumplan satisfactoriamente las demandas de los ingenios, y se maximice el balance energético total del sistema completo. Para cumplir este objetivo se utilizó un enfoque basado en Sistemas Multiagentes (SMA), y se comparó la performance del sistema con la obtenida por el método Simplex desarrollado en un trabajo anterior [3].

\subsection{Una visión de agentes}

El problema planteado es de gran tamaño (se estiman más de 300.000 parcelas para los 15 ingenios existentes), y presenta una cantidad de variables y restricciones operativas. Se optó por un enfoque orientado a SMA debido a las facilidades que este paradigma ofrece respecto a escalabilidad y paralelización. Independientemente del tamaño del problema, los SMA resultan muy simples de paralelizar, lo que permite encarar con facilidad problemas de gran tamaño en poco tiempo. Por otro lado, agregar nuevas variables y comportamientos al sistema no presenta grandes dificultades, a diferencia de otras técnicas como Simplex que pueden no ser capaces de seguir atacando el problema si una de las nuevas restricciones o variables introducidas no resulta lineal [17].

Dentro del modelo adoptado, cada parcela está representada por un agente parcela y similarmente los ingenios por un agente ingenio. El sistema desarrollado está basado en el concepto de Fuerzas de Atracción [12-13] para modelar el comportamiento de los distintos agentes. De esta manera, un agente ingenio genera una determinada atracción sobre todos los agentes parcela. Luego, cada agente parcela se encarga de evaluar cuál de todos los agentes ingenio resulta más atractivo, y comunica su decisión al agente ingenio seleccionado, informándole además su producción. A continuación, cada agente ingenio recibe los datos de todos los agentes parcela que lo seleccionaron, evalúa si cumple con sus requerimientos de demanda y en función de ello, genera una nueva atracción. Esta nueva atracción es enviada a todos los agentes parcela y el ciclo se repite hasta encontrar una situación de equilibrio, en donde los agentes parcela no modifiquen su decisión del agente ingenio seleccionado, y los agentes ingenio no modifiquen su atracción sobre los agentes parcela. Este comportamiento se muestra en el diagrama de la Figura 1. 


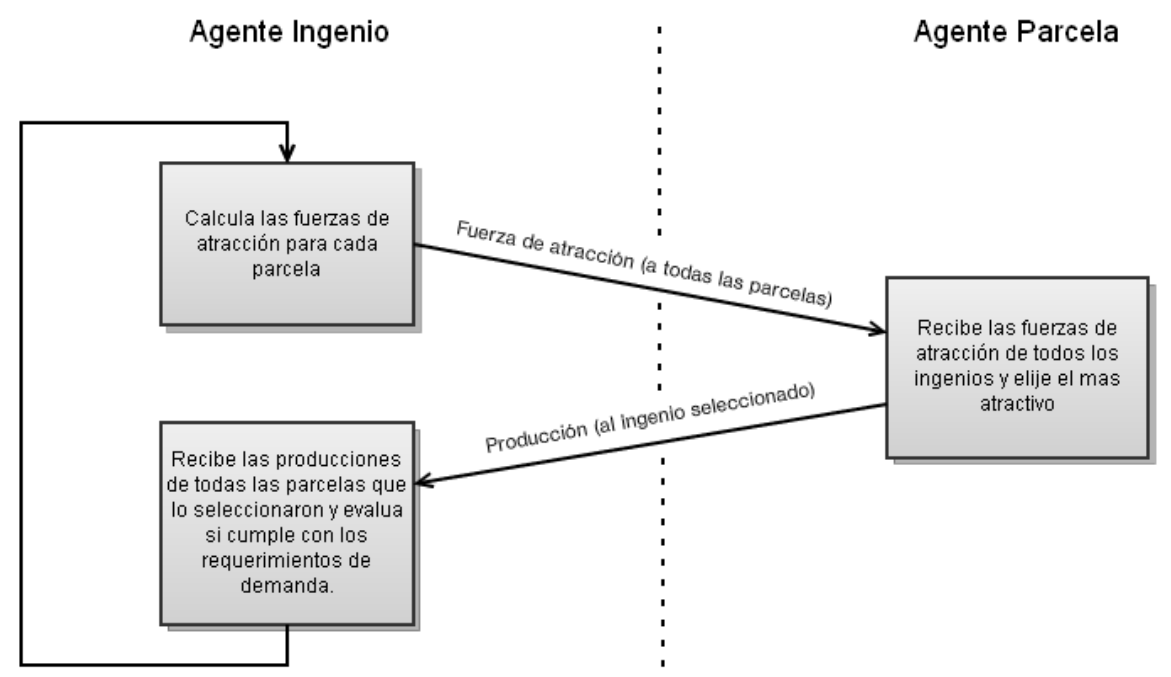

Fig. 1. Interacción entre los agentes ingenio y los agentes parcela.

De esta forma los conceptos de agente parcela y agente ingenio resultan independientes entre sí, lo cual facilita la escalabilidad del modelo tanto en tamaño del problema como en complejidad del modelo matemático subyacente, y la paralelización de la implementación.

\subsection{Modelo Matemático Utilizado}

Como ya mencionamos, el modelo utilizado en el presente trabajo está basado en el concepto de Fuerzas de Atracción, donde los agentes ingenios ejercen fuerzas, y los agente parcelas reaccionan de acuerdo a la fuerza total ejercida sobre ellos, siendo el objetivo maximizar el Balance Energético Total del sistema. Se presentan a continuación la definición formal de los conceptos mencionados.

El Balance Energético $\left(B E_{i j}\right)$ total para transportar la producción de una parcela $i$ hacia el ingenio $j$ puede expresarse como:

$$
B E_{i, j}=\frac{B E P_{i} \cdot B E I_{j}}{C_{i, j}} \quad \forall i=1, \ldots, m, j=1, \ldots, n
$$

donde $B E P_{i}$ es el Balance Energético de la parcela $i, B E I_{j}$ es el Balance Energético del ingenio $j$ y $C_{i, j}$ es el costo (medido como balance energético) de transportar la producción desde la parcela $i$ hasta el ingenio $j$. Luego, el Balance Energético Total $\left(B E_{T}\right)$ del sistema puede expresarse como: 


$$
B E_{T}=\sum_{i=1}^{m} \sum_{j=1}^{n} B E_{i j} \cdot \operatorname{sol}(i, j)
$$

donde $\mathrm{sol}$ es un estado del sistema en una iteración, y consiste en una matriz de $m$ filas y $n$ columnas, en la que cada elemento $\operatorname{sol}(i, j)$ de la matriz es igual a 1 si el agente parcela $i$ selecciona al agente ingenio $j$, y cero en cualquier otro caso.

Entonces, el objetivo del sistema actual es encontrar una solución tal que maximice $B E_{T}$, cumpliendo con la siguiente restricción:

$$
\operatorname{Pmin}_{j} \leq \sum_{i=1}^{m} P_{i} \cdot \operatorname{sol}(i, j) \leq \operatorname{Pmax}_{j} \quad \forall j=1, \ldots n
$$

donde $\operatorname{Pmin}_{j}$ es la producción mínima que necesita el ingenio $j$ para funcionar, $\operatorname{Pmax}_{j}$ es la producción máxima de la que es capaz el ingenio $j, P_{i}$ es la producción de la parcela $i$ y $\operatorname{sol}(i, j)$ es la solución del sistema ya definida.

La metodología de solución propuesta presenta un comportamiento que es característico de los sistemas emergentes [18], por lo que no es posible obtener de manera exacta el valor máximo de $B E_{T}$. Por tal motivo, se utiliza una heurística basada en el concepto de fuerza de atracción que permita obtener soluciones aproximadas al ideal con un margen de error razonable.

Como mencionamos anteriormente, cada agente ingenio genera una fuerza de atracción sobre los agentes parcela. La fuerza de atracción de un agente ingenio $j$ sobre un agente parcela $i, F A_{i j}$, se define de la siguiente manera:

$$
F A_{i, j}=\frac{C_{i, j} \cdot K_{j}}{B E I_{j}} \quad \forall i=1, \ldots, m, j=1, \ldots, n
$$

donde $i$ representa un agente parcela, $j$ representa un agente ingenio, $B E I_{j}$ es el balance energético del ingenio $j, C_{i, j}$ expresa el costo de transportar la producción desde la parcela $i$ hasta el ingenio $j$, y $K_{j}$ es el factor de atracción del ingenio $j$.

Este factor de atracción $\mathrm{K}$ tiene como principal objetivo controlar la fuerza de atracción de un agente ingenio. Resulta necesario evitar que un agente ingenio muy atractivo acumule demasiados agentes parcela (superando la capacidad de producción del ingenio), e inversamente, que un agente ingenio poco atractivo no sea seleccionado por suficientes agentes parcela (implicando el cierre efectivo del ingenio). Así, si la suma de la producción de todos los agentes parcela que eligieron a un agente ingenio no alcanza a cubrir un valor mínimo de demanda del ingenio, el factor de atracción incrementa la fuerza de atracción de ese agente ingenio. Contrariamente, si la suma de la producción de todos los agentes parcela que eligieron a ese agente ingenio supera la capacidad máxima del ingenio, entonces el factor de atracción disminuye la 
fuerza de atracción del agente ingenio, con la intención de que resulte menos atractivo para los agentes parcela, y que algunos de éstos decidan ir hacia otro agente ingenio.

Este comportamiento del factor de atracción $\mathrm{K}$ se ve reforzado si la situación indeseada se mantiene en el tiempo, con el fin de forzar la convergencia del sistema. Este factor de atracción resulta crítico tanto en el cumplimiento de las restricciones como en la forma y valor en que el sistema se estabiliza.

Luego, cada agente parcela recibe las fuerzas de atracción calculadas de todos los agentes ingenio, y en base a ellas decide cuál es el más atractivo. Para tomar dicha decisión, cada agente parcela $i$ calcula un Factor de Evaluación $F E_{i, j}$ para cada agente ingenio $j$, definido en la siguiente fórmula:

$$
F E_{i, j}=F A_{i, j}+\frac{B E P_{i}}{\operatorname{mean}\left(\left\{B E P_{k}: \operatorname{sol}(k, j) \neq 0,1 \leq k \leq m\right\}\right)} \forall i=1, \ldots, m, j=1, \ldots, n
$$

donde $F A_{i, j}$ es la fuerza de atracción que ejerce el agente ingenio $j$ sobre el agente parcela $i, B E P_{i}$ es el Balance Energético de cada agente parcela $i$, y el valor en el cociente indica el promedio de los balances energéticos de los agentes parcela que seleccionaron en el paso anterior al agente ingenio $j$. El segundo término es mayor que 1 si el agente parcela presenta un rendimiento superior al promedio de los agentes parcela que seleccionaron en el paso anterior a ese agente ingenio, y menor que 1 en caso contrario. El efecto neto es que el agente ingenio atrae agentes parcela con mejor rendimiento y repele agentes parcela con bajo rendimiento.

Finalmente, se toma como valor inicial del sistema una solución ad-hoc, quasióptima por construcción (desde el punto de vista del Balance Energético Total del sistema), donde cada parcela entrega su producción al ingenio que le resulta más conveniente. Esta solución en la mayoría de los casos no cumpliría con las restricciones mencionadas, pero proporciona un buen punto de partida cercano al óptimo teórico para la búsqueda del SMA, entregando buenos resultados con muy pocas iteraciones en todos los ejemplos estudiados.

\subsection{Comparación con Algoritmo Simplex}

En cuanto a la comparación con el algoritmo Simplex en grafos, utilizado en [3], la función energía descripta en (2) no cumple las hipótesis necesarias para el correcto funcionamiento del algoritmo Simplex (ver [17], [3]). Se procede a simplificar el modelo de forma tal de poder aplicar ambos algoritmos al mismo problema y comparar su funcionamiento. El sistema Multiagentes fue modificado para optimizar la función:

$$
F_{\text {comp }}(\text { sol })=\sum_{i=1}^{n} \sum_{j=1}^{p} C_{i, j} \cdot \operatorname{sol}(i, j) \cdot P_{i}
$$


donde $P_{i}$ es la producción total en toneladas de la parcela $i$. Así, dentro del modelo SMA, la fuerza de atracción del agente ingenio $j$ sobre el agente parcela $i$ será:

$$
F A_{i, j}=C_{i, j} \cdot P_{i} \cdot K_{j} \quad \forall i=1, \ldots, m, j=1, \ldots, n
$$

Y el factor de evaluación del agente parcela $i$ para el agente ingenio $j$, es directamente:

$$
F E_{i, j}=F A_{i, j}
$$

Este modelo es claramente más simple que el anterior, por lo que se estima que la performance de ambos sistemas puede ser comparada sobre la base de este modelo en distintos ejemplos.

\section{$4 \quad$ Resultados Obtenidos}

El presente trabajo presenta un modelo matemático del problema y un primer prototipo del sistema de optimización planteado. Este prototipo fue probado utilizando varios problemas diferentes, creados sintéticamente sobre la base de la situación esperada en el problema real. El primer caso fue el utilizado en [3], es un modelo simple que cuenta con 6 ingenios y 245 parcelas, modificado para poder estimar a priori los resultados correctos. Se utilizaron además 4 ejemplos de tamaño creciente generados en forma aleatoria, de $1.000,2.000,3.000$, y 30.000 parcelas con 15 ingenios cada uno, y se probaron ambos sistemas con estos ejemplos. Se presentan en primer lugar los resultados de todos los ejemplos obtenidos por el Sistema Multiagentes corriendo en su versión estándar (maximizando la energía definida al comienzo de la sección 3.3). A continuación se muestran los resultados comparados entre el Sistema Multiagentes simplificado y Simplex para todos los ejemplos mencionados. Es importante destacar que el ejemplo de 3.000 parcelas tomó en Simplex 4.84 horas para completarse, y el ejemplo de 30.000 no había terminado luego de 12 horas de proceso, por lo que fue detenido. De acuerdo a la curva presentada más adelante en la figura 5 , se estima un tiempo de cálculo superior a los 6 meses. No se utilizaron ejemplos de mayor orden de magnitud de parcelas puesto que su ejecución en Simplex en una máquina común resultan inviables, y la codificación actual del Sistema Multiagentes no permite manejar matrices mayores a 65.000 parcelas.

\subsection{Sistema Multiagentes}

En la figura 2 se presentan los valores de Energía en función de las iteraciones para el primer ejemplo utilizando Sistemas Multiagentes con la función de optimización descripta inicialmente, y en la figura 3 se presentan los valores de porcentaje de demanda satisfecha de cada ingenio, en función de las iteraciones. Estas figuras muestran cómo la solución inicial se construye de manera de optimizar el Balance Energético Total del sistema (fig. 2) pero sin tener en cuenta las restricciones de producción 
de los ingenios (fig. 3). El sistema modifica esta situación de manera de llevar las entregas a la zona deseada y cumplir las restricciones.

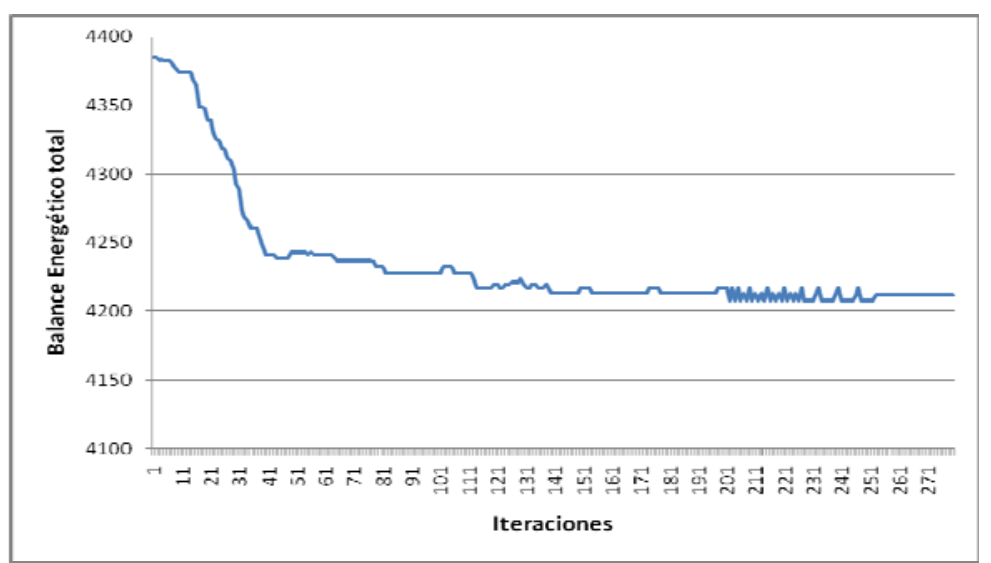

Fig. 2. Variación de la energía total del sistema para el primer ejemplo

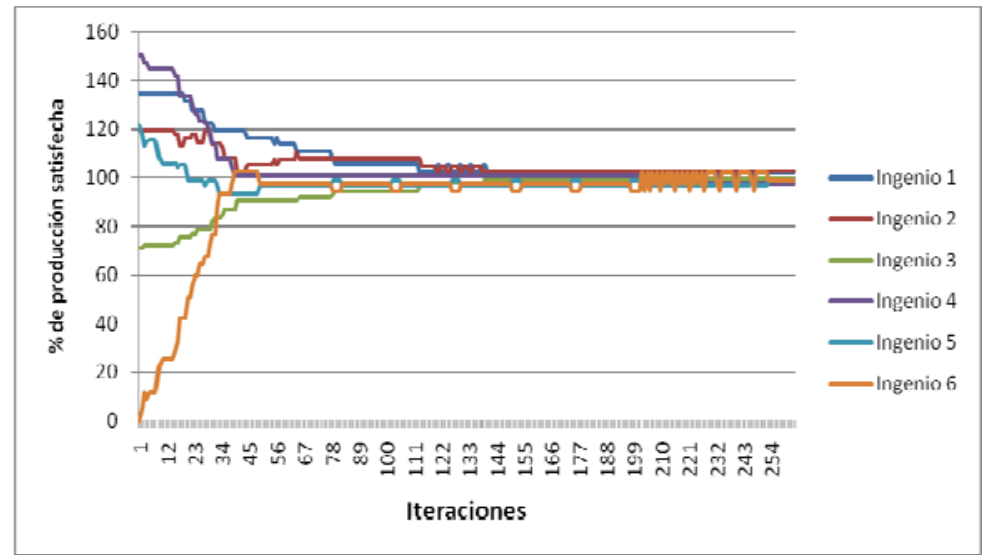

Fig. 3. Porcentaje de producción satisfecha de cada ingenio para el primer ejemplo

En la figura 4 se observan los valores de Energía en función de las iteraciones de los otros 4 ejemplos al cabo de 110 iteraciones, presentadas en forma normalizada. En esta gráfica puede apreciarse, además del comportamiento característico ya descrito, que para ciertas configuraciones la energía oscila entre un rango de valores acotado, sin establecerse en una solución determinada. Como la variabilidad de la solución ocurre con una cota menor que $0.5 \%$ puede considerarse que cualquier solución que se encuentre dentro de este rango es igualmente satisfactoria. 


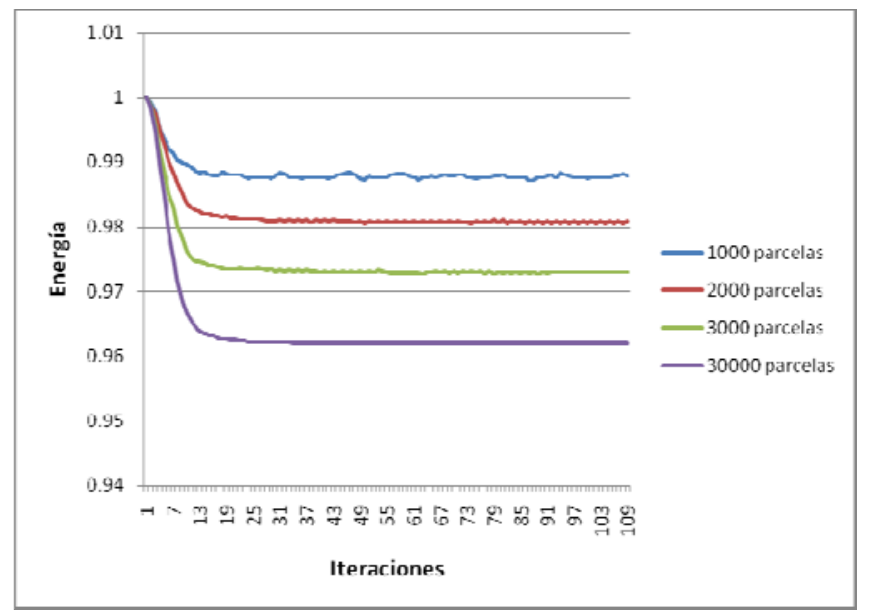

Fig. 4. . Variación de la energía del sistema para los ejemplos de 1000, 20003000 y 30000 parcelas con 15 ingenios normalizadas

En los ejemplos se considera la capacidad máxima de procesamiento de los ingenios $(100 \%$ de su capacidad, con una variabilidad del 3\%). Se consideró además que la producción total de caña coincide con la demanda colectiva de los ingenios. Estas dos consideraciones se realizan para poder aplicar el sistema basado en Simplex desarrollado en [3]. El Sistema Multiagentes permite la modificación del porcentaje de capacidad óptima y de variabilidad, así como también manejar situaciones en las que la producción total de caña es distinta a la demanda colectiva de los ingenios.

Con todos los experimentos realizados, se pudo comprobar que el modelo se ajusta de manera adecuada a lo esperado.

\subsection{Comparación con Simplex}

Se compararon los comportamientos de los dos sistemas en los ejemplos descritos, sobre la base del modelo descripto en la sección 3.4. En la figura 5 puede observarse como cambia el tiempo de ejecución en función de la cantidad de parcelas para el Sistema Multiagentes (fig. 5a) y para el método Simplex (fig. 5b).

Es importante destacar que el sistema Simplex es secuencial y está programado en MATLAB $^{\circledR}$, mientras que el Sistema MultiAgentes es esencialmente paralelo y está programado en Java SE, por lo que la comparación se realiza en función del crecimiento del tiempo de ejecución con el aumento del problema, siguiendo [19]. 

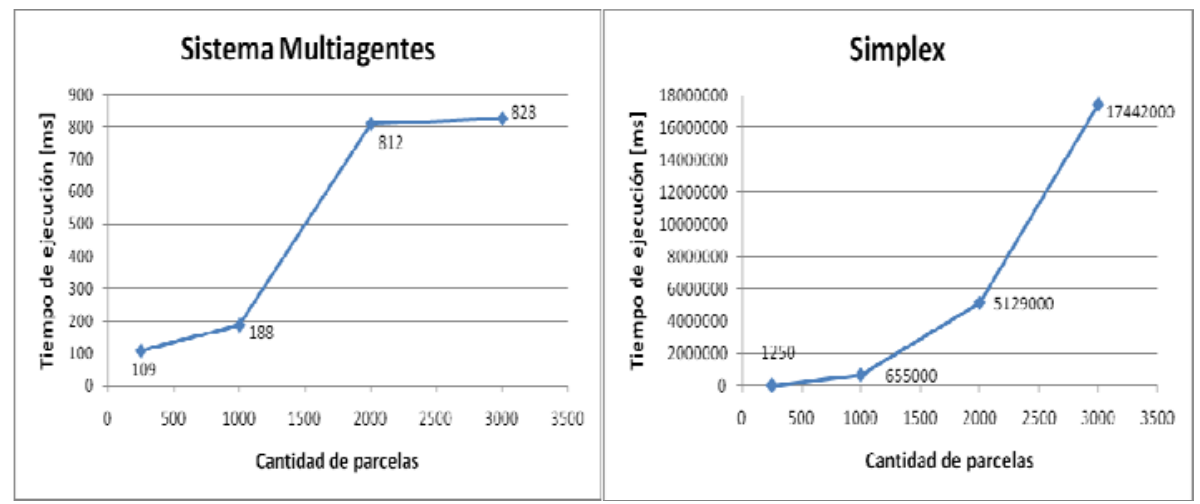

Fig. 5. Tiempos de ejecución en milisegundos para (a) SMA y (b) Simplex.

Se observa en el gráfico de la figura 3 una fuerte tendencia creciente en el Simplex que no se observa en el Sistema Multiagentes. Inclusive, se puede apreciar que existe una diferencia de cinco órdenes de magnitud a favor de este último, tendencia que se acentúa con el tamaño del problema.

En la figura 6 puede apreciarse una comparación entre los valores de optimización alcanzados por el Simplex y aquellos alcanzados por el Sistema Multiagentes para los ejemplos de comparación propuestos.

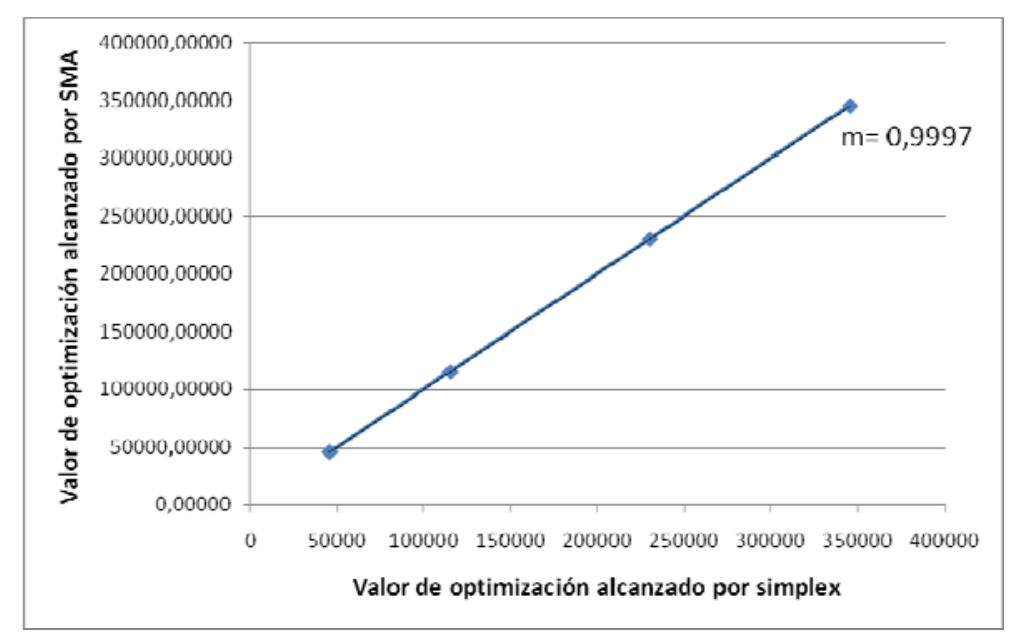

Fig. 6. Comparación de los resultados obtenidos para los ejemplos con 245, 1000, 2000, y 3000 parcelas

Se puede apreciar en la figura 6 que el valor final de la función objetivo obtenido por ambos sistemas es el mismo en todos los casos probados. Además, para uno de los ejemplos (para el que podía predecirse una solución a priori), se comparó las soluciones encontradas de los dos sistemas. Se encontró que el valor final obtenido de ambos 
sistemas coincide con un error menor al $0.3 \%$. En cuanto a la solución obtenida, se observa que el $90 \%$ de las parcelas tienen el mismo destino en ambas soluciones. Esto resulta razonable si se tiene en cuenta que la solución no es única, dado que cualquier permutación de las parcelas menos eficientes entre ingenios cercanos no resultará en cambios significativos en la función. Se observa, tal como podría predecirse, que este $10 \%$ de las parcelas que tienen destino diferente representa un cambio de valor de la función de tan solo un $0.3 \%$.

\section{Conclusiones y líneas futuras de investigación}

Se desarrolló y probó en este trabajo un sistema basado en Sistemas Multiagentes para la optimización del transporte de caña de azúcar. Los resultados obtenidos muestran que el sistema resuelve satisfactoriamente el problema en los casos probados, proporcionando resultados similares a los obtenidos por programación lineal [3] con una performance mucho más alta.

Los resultados pueden resumirse de la siguiente manera:

- En el caso del modelo matemático simplificado, el valor final obtenido para la función de optimización es similar al de Simplex (la diferencia es menor al 1\%). En el caso del modelo matemático completo, los resultados del Sistema Multiagentes concuerdan con lo esperado.

- El tiempo de ejecución del Sistema Multiagentes es varios órdenes de magnitud menor que el de Simplex para todos los ejemplos probados. Además, la tendencia de crecimiento de Simplex es mucho mayor.

- Si bien los resultados obtenidos fueron satisfactorios, la convergencia del sistema aún es muy sensible a los parámetros del factor de atracción K, siendo necesario encontrar una solución más estable.

- Resulta imposible aplicar Simplex en el caso de restricciones no lineales (como en el caso del Modelo Matemático completo utilizado en este trabajo). En cambio, los sistemas Multiagentes son fácilmente escalables, en el sentido de que es sencillo no solo paralelizarlos, sino agregar nuevas variables y restricciones del problema.

- El sistema en su forma presente puede aplicarse a muchas agroindustrias en diversas zonas del país, ya que las características planteadas hasta el momento son generales.

- Si bien el sistema actual no permite abordar problemas grandes (más de 65.000 parcelas) esto no representa un problema dada la capacidad de los sistemas multiagentes de ser paralelizables.

El sistema propuesto en este trabajo representa la parte inicial de un proyecto a largo plazo para la optimización del transporte de caña de azúcar en la provincia de Tucumán. El modelo matemático sobre el que se basa el sistema es un modelo simplificado, que contiene las variables y restricciones principales y simula adecuadamente el problema, pero debe ser complejizado para tener en cuenta otras restricciones operativas (como por ejemplo el tipo y variedad de la caña, el período completo de la zafra, etc.). 
En cuanto a líneas futuras de investigación, se pueden mencionar las siguientes:

- Algunas de las restricciones planteadas provienen de la industria (como las demandas de los ingenios, y la decisión de restringir la optimización a un día fijo), pero otras condiciones como la cantidad de caña disponible en relación con la demanda colectiva de los ingenios, si bien contemplada en alguna medida en ambos sistemas, no fue presentada en detalle debido a que es una característica crítica para la aplicabilidad del sistema. Será estudiada en mayor profundidad y validada contra datos industriales en futuros trabajos.

- Debe desarrollarse un sistema de llenado de la matriz de costos que tenga en cuenta las diversas variables del problema, incluyendo estado de los caminos y costo en combustible.

- El sistema debe complejizarse para incluir otras características de la caña de azúcar que resultan importantes para el problema (como variedad de la caña, estimado de porcentaje de fibra, etc.).

- Se deben incorporar al sistema las características y demandas propias de la industria de azúcar de caña de la provincia de Tucumán (forma de trabajo, características de la producción, vehículos existentes, etc.).

\section{Agradecimientos}

Los autores agradecen el apoyo del proyecto PID 1318 de la UTN y del proyecto CIUNT 26/E457, y a la EEAOC el apoyo, contacto con la industria, y uso de los datos.

\section{Referencias}

1. Lannes, A; Pucci, C; "La agroindustria azucarera y su influencia en el PBI de Tucumán", Banco Santiago del Estero, 2007

2. Pérez, D; Fandos, C; Scandaliaris, J; Mazzone,L; Soria,F; Scandaliaris, P; "Estado actual y evolución de la productividad del cultivo de caña de azúcar en Tucumán y el noroeste argentino en el período 1990-2007"; EEAOC Publicación Especial 34, 2007.

3. Diez, O; Will, A.; Rodriguez, S.; et.al.: "Optimización del Transporte de Caña de Azúcar desde el productor al ingenio en la provincial de Tucumán", Actas del primer Taller de Matemática Industrial, TAMI 2010, fascículo 6, serie B, Cursos y Seminarios en Matemática, FCEyN-UBA. 2010.

4. Giles, R., Lyne P., Venter R., Van Niekerk J., \& Dines G.: "Vehicle Scheduling Project Success At South African And Swaziland Sugar Mills", Proc S Afr Sug Technol Ass (2009) 82: $151-163$

5. Giles, R., Dines, G., Lyne, P., Bezuidenhout, C.: "The complexities of introducing the complexities of introducing the FREDD vehicle scheduling system into the Darnall mill area", Proc. South African Tech. Ass., 80 (2006), 66-70

6. Jorio, R., Legendre, B., Gautz, L., Abdellaoui, R.:"Simulation model to reduce the impact of rain stops and breakdowns on sugar harvesting, thansport, and crushing system performances", Proc. South African Tech. Ass., 80 (2006), 115-119 
7. Le Gal, P., Le Masson, J., Bezuidenhout, C., Lagrange, L.: "Coupled modelling of sugarcane supply planning and logistics as a management tool”, Comput. Electron. Agric. 268 (2009), 168-177

8. Lopez Milán, E., Miquel Fernandez, S., \& Plá Aragonés, L.: "El Problema Del Transporte De La Caña De Azúcar En Cuba”, Investigación Operacional 252 (2004), 148-157

9. Salles Scarpari, M., \& Gomes Ferreira, E.: "Optimized Agricultural Panning of Sugarcane using Linear Pogramming”, Investigacion Operacional 312 (2010),126-132 (BRASIL)

10. Gen, M., Chen, R., Lin, L.: "Network Models and Optimization: A Multiobjective Genetic Algorithms Approach", Springer Decision Engineering Series, Springer, 2008

11. Syarif A., Gen, M.: "Solving and extended logistics model using hybrid genetic algorithms", 11th Asian Pacific engineering and management systems conference, 2010.

12. Zhao D; Yi J.: "Robot Planning with Artificial Potential Field Guided Ant Colony Optimization Algorithm", Advances in Natural Computation, Springer,2006

13. Chen B; Cheng H:" A Review of the Applications of Agent Technology in Traffic and Transportation Systems", IEEE Transactions On Intelligent Transportation Systems, Vol. 11, No. 2, Junio 2010

14. Fox, M., Babuceanu, M., and Teigen,R.: "Agent-Oriented Supply Chain Management”, International Journal of Flexible Manufacturing Systems, 12(2000), 165-188

15. Pardoe, D., and Stone, P.: "An Autonomous Agent for Supply Chain Management", Handbooks in Information Systems Series, Business Computing, Emerald Group, 2009

16. Li, Ling: "Supply Chain Management: An evolutionary View", Chapter 1 in "Supply Chain Management: Concepts, Techniques, and Practices", World Scientific Publishing Co., 2007

17. Bazaraa, M., Jarvis, J., Sherali, H.: "Linear Programming and Network Flows", 3rd ed., Wiley Interscience, 2005.

18. Johnson, Steven: "Emergence: The connected lives of ants, brains, cities, and software", Scribner, 2003

19. Aho, A., Hopcroft, J., and Ullman, J.: "Data structures and Algorithms", 2nd ed., Addison Wesley, 1987. 